\title{
ROBOTIC MOUNTING SYSTEM FOR LARGE-PANEL BUILDING
}

\author{
Thomas Bock*, Alexej Bulgakow**, Dimitry Parshin***, Sergej Tkachev ${ }^{* * *}$ \\ * Technical University Munich, Germany, thomas.bock@bri.ar.tum.de \\ ** South Russian State Technical University, Russia a.bulgakow@gmx.de \\ *** Rostov State Building University, Russia, d.parshin@mail.ru
}

\begin{abstract}
The paper presents the principles of construction and structural organization of the robotic mounting system. The concept of developing models of manipulators with elastic couplings has been suggested in terms of which we have obtained mathematical description of the crane. The problems of constructing mathematical models of the system manipulators interaction have been considered and the technique for calculating moments to control the mounting system has been suggested.
\end{abstract}

Keywords: construction, large-panel building, mounting, robotization.

\section{INTRODUCTION}

The promising trend in automation of bearing-wall buildings mounting is the creation of robotic mounting system with interactive and adaptive control. In the process of mounting wall panels there appears the problem of wall panels positioning and retaining panels on the design position during its installation and fixing. Incorporation into the system of the mounting robot makes it possible to mechanize and automate the process of the structures orientation and mounting.

\section{PRINCIPLE OF THE MOUNTING SYSTEM ONSTRUCTION}

The basis of the mounting system incorporates a tower crane and a positioning robot with a mounted wall panel. The functions are distributed between the crane and the robot in the following way. The crane supplies the panel to the place of mounting and keeps it during the process of mounting and orientation and the positioning robot performs a panel space orientation before mounting. The crane and the robot interaction in the zone of a panel mounting is so organized that the crane doesn't interfere free movement and turning of the plate and the robot ensures positioning and orientation without any significant loads on the manipulating system.

Structural organization of the robotic mounting system is presented in fig. 1 . The tower crane being incorporated into the system is equipped with a special automated cross-piece and a system of programmed control (SPC). On the basis of control vector $\mathrm{g}_{c}, \dot{\mathrm{g}}_{c}$ and current position of joint coordinates $\mathrm{q}_{c}$ and joint speed $\dot{\mathrm{q}}_{c}$ the crane control system produces control forces and moments $\mu_{c}$. The mounting robot is equipped with two manipulators and a moving platform. The investigations have shown that application of two manipulators ensures a reliable joining with a panel and fulfillment mounting operations at rather small static and dynamic loads on the manipulating system. The mounting robot is equipped with the system of programmed control with elements of adaptation. The main component of the whole system is the data-measuring system, its function is to control position and orientation of the mounted panel and also the reference of the robot platform to the design axes of the erected object. The base of the DMS is the laser set-point devices and photo-matrix transducers located on the cross-piece and on the manipulators' grippers. DMS forms the system state vector $\mathrm{X}_{p}$ on the basis of which the synchronization and control device (SCD) of the upper level performs planning of the system mechanisms movements.

A 3-level control system is implemented in the whole system. The synchronization and control device of the upper level on the basis of the planning algorithms forms control vectors $\mathrm{g}_{r}, \dot{\mathrm{g}}_{r}$ for the robot system control and the vectors $\mathrm{g}_{c}, \dot{\mathrm{g}}_{c}$ for the crane control system. And the vector formation is effected with account of the wind load and elastic deformations. Taking into consideration complexity and responsibility of the system's mechanisms operation, planning of the crane and the robot's movements is carried out on the basis of simulation of the mounting process. The system models used for this purpose are designed taking into account the distribution of functions that are carried out by the 


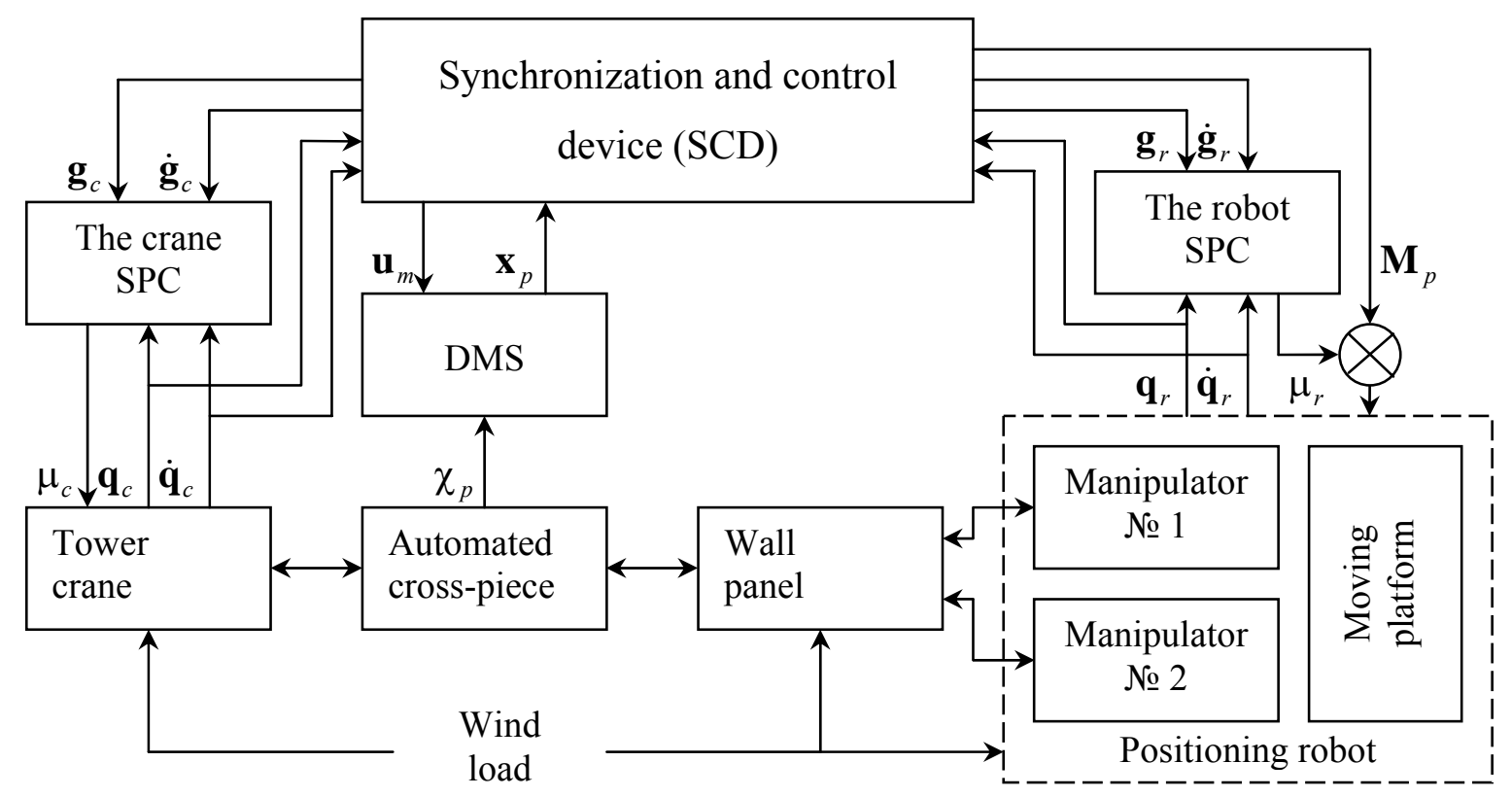

Fig. 1.

crane and the mounting robot. During the process of a panel positioning and mounting strict requirements are not specified to the crane relative to dynamic characteristics and the robot is to provide high accuracy of positioning. Therefore the crane control is realized with the help of kinematic algorithms according to the designed trajectory. The requirements of high accuracy of the mounting robot positioning and a wide range of loads changes of the manipulator drive cause the necessity of applying dynamic control laws for the robot control. Besides on the upper level we calculate the joint moments necessary to overcome the panel resistance $\mathrm{M}_{p}$; on the medium level - the moments stipulated by the dynamic properties of each manipulator on its own $\mu_{r}$.

\section{ATHEMATICAL MODEL OF THE MOUNTING SYSTEM}

A specific feature of developing dynamic model of the robotic system is the availability of branched and closed architectures. So the application of classical methods is connected with certain hardships. When using the modified Lagrange - Euler method the analytical representation of the system becomes awkward and is not convenient for investigation. Application of the Newton method requires finding minimum of functions that is not efficient from the computational point of view and not convenient for analytical investigation. Therefore mathematical model for the mounting system constructed according to the principles of decomposition we have obtained tree autonomous mechanical systems the interaction between which is accounted according to the principle of small motions. The essence of this principle lies in that before the simulation begins the linked bodies are in the state of rest and don't influence each other. Having begun their movement the bodies carry out small motions according to which the displacements are determined. The arising elastic deformation cause reaction forces in each point of the robot attachment:

$$
\begin{aligned}
& \mathrm{N}^{(k)}=(\delta+\gamma \cdot s)\left(\mathrm{x}_{r}^{(k)}-\mathrm{x}_{c}^{(k)}\right), k=\overline{1, l}, \\
& s \equiv d / d t
\end{aligned}
$$

where $\delta, \gamma$ are the coefficients of stiffness and connection dissipation; $\mathbf{x}_{r}^{(k)}, \mathbf{x}_{c}^{(k)}$ are the vectors $(3 \times 1)$ of Cartesian coordinates of the $k$-th suction cup and the places of this attachment.

Therefore the model of the mounting system consists of 3 channels simulating the dynamics of the robot and the crane manipulators. The interacting force taking place between them is determined by the formula (1).

Mathematical description of the robot manipulators is carried out on the basis of Denavite-Hartenberg transformation. Vector $\mathbf{X}_{r}^{(k)}$ is connected with the manipulator joint coordinate by the equation

$$
\mathbf{x}_{r}^{(k)}=T_{r}\left(\mathrm{q}_{r}\right) \cdot \mathrm{d} \mathbf{x}_{r}^{(k)},
$$

where $T_{r}\left(\mathrm{q}_{r}\right)$ is the transition matrix from the absolute system of coordinates to the gripper system of coordinates; $\mathrm{q}_{r}$ is the manipulator joint coordinates; $\mathrm{dx}_{r}^{(k)}$ are the displacement of the $\mathrm{k}$-th suction cup in the gripper coordinate system. 
The dynamics of each manipulator is described by the Lagrange-Euler equation

$$
\mathrm{M}=D(\mathrm{q}) \cdot \ddot{\mathrm{q}}+\mathrm{h}(\mathrm{q}, \dot{\mathrm{q}})+\mathrm{c}(\mathrm{q})+\sum_{k=1}^{l} \mathrm{M}_{b}^{(k)},
$$

where $\mathrm{M}$ is the vector of joint forces created by the power drives in the manipulators joints; $D(\mathrm{q})$ is the symmetrical matrix of dynamics with dimensionality of $n \times n ; \mathrm{h}(\mathrm{q}, \dot{\mathrm{q}})$ is the vector $(n \times 1)$ of Coriolis and centrifugal forces; $\mathbf{M}_{b}^{(k)}$ is the vector $(n \times 1)$ of external forces; $n$ is the number of joint coordinates

Vector $\mathbf{M}_{b}^{(k)}$ is stipulated by the influence of communication forces $\mathrm{N}_{k}$ and is connected with them by the ratio

$$
\begin{aligned}
& \mathrm{M}_{b}^{(k)}=J_{n}^{\mathrm{T}}\left(\mathrm{q}, \mathrm{dx}^{(k)}\right) \cdot \mathrm{N}^{(k)}, \\
& \left(J_{m}(\mathrm{q}, \mathrm{dx})\right)_{j, i}=\left(U_{i, m}(\mathrm{q}) \cdot \mathrm{dx}\right)_{j}, \\
& j=\overline{1,3}, i=\overline{1, m},
\end{aligned}
$$

where $U_{i, m}(\mathrm{q})$ is the partial derivative of the transition matrix from the base coordinate system to $m$ coordinate system with respect to the $i$-th joint coordinate.

Construction of the tower crane model is performed with account of elastic deformations in the crane design that arise under the influence of wind and dynamic loads. The model is constructed for movements at low speeds when the amplitude of the wire rope vibrations doesn't achieve great values. In this case wave processes in the rope can be neglected. The adopted limitations for the vibration amplitude are usually carried out on performing the final phase of the panel mounting.

On the basis of the analysis of the crane possible motions with the attached wall panel we have developed an equivalent mechanical diagram of the manipulator. According to the mechanical diagram vector $\mathbf{X}_{c}^{(k)}$ is connected with joint coordinates of the crane with the equation

$$
\mathrm{x}_{c}^{(k)}=T_{c}\left(\mathrm{q}_{c}\right) \cdot \mathrm{dx} \mathrm{x}_{c}^{(k)},
$$

were $T_{c}\left(\mathrm{q}_{c}\right)$ is the matrix of transition from the system of coordinates to the panel system of coordinates; $\mathrm{q}_{c}$ are joint coordinates of the crane; $\mathrm{dx}_{c}^{(k)}$ is the displacement of the attachment of the $k$ th suction cup which is measured in the panel system of coordinates.
The account of elastic forces in the axes of motion is convenient to carry out by the way of introducing additional elastic elements (springs) the rigidity of which is equivalent to the rigidity of the elastic body in direction of the axis of motion movement. Taking into account that the time constant of the drives of the crane axes of motion is by an order less than the links' period of vibration and the drives don't have static mistake then the crane driving force is equal to the sum elastic and dissipative forces arising because of the error in current and stable values of joint coordinates

$$
\begin{aligned}
& M_{c}^{(i)}=\left(g_{c}^{(i)}-q_{c}^{(i)}\right) \cdot \alpha_{i}+\left(\dot{g}_{c}^{(i)}-\dot{q}_{c}^{(i)}\right) \cdot \beta_{i}, \\
& i=\overline{1, n}
\end{aligned}
$$

where $g_{c}^{(i)}, \dot{g}_{c}^{(i)}$ are non-deformed positions and velocity of the $i$-th joint coordinate; $\alpha_{i}, \beta_{i}$ are the coefficients of rigidity and dissipation of the fictitiously introduced spring which coincides in direction with the $i$-th joint coordinate and the nondeformed state of the controlled coordinates is determined by the angle and rotational speed of the stable position of the additive axes of motion imitating elastic connections are taken as equal to zero.

During the process of mounting the crane may experience substantial influence of the wind load therefore we introduce into the equation (2) a summand taking account of that influence. The concentrated force equivalent to the wind load acting the $i$-th link will be written in the form

$\mathrm{F}_{w}^{(i)}=\left(\vartheta_{x}^{(i)}(\mathrm{q}) P_{x} \quad \vartheta_{y}^{(i)}(\mathrm{q}) P_{y} \quad \vartheta_{z}^{(i)}(\mathrm{q}) P_{z}\right)^{\mathrm{T}}$, $i=\overline{1, n}$,

where $\vartheta_{x}^{(i)}(\mathrm{q}), \vartheta_{y}^{(i)}(\mathrm{q}), \vartheta_{z}^{(i)}(\mathrm{q})$ are the functions of the unit wind load of the $k$-th link in the direction of the axes of the base coordinate system; $P_{x}, P_{y}$, $P_{z}$ is the distribution of the wind flow along the axes of the absolute coordinate system. The obtained force is converted from the Cartesian coordinates to the joint ones

$$
\mathrm{M}_{w}^{(i)}=J_{i}^{\mathrm{T}}\left(\mathrm{q}, \mathrm{r}_{w}^{(i)}(\mathrm{q})\right) \cdot \mathrm{F}_{w}^{(i)},
$$

$\mathbf{r}_{w}^{(i)}(\mathbf{q})$ is the displacement vector $(3 \times 1)$ for the concentrated force point $\mathrm{F}_{w}^{(i)}$. On the basis of the equations (2-5) we can plot a block diagram of the mounting system model that is presented on the fig. 2 where $G$ is the function determining dynamics of the mechanical part of the manipulator.

The mounting system control provides 2 modes interactive and automatic. Interactive control is used 
at the stage of grasping a panel by the positioning robot when its position is arbitrary and it is difficult to perform joining operations. In this mode an operator with the help of 2 special-purpose handles carries out control of the robot manipulators moving the gripper device so as to join it with the panel. In the automatic mode the process of a panel orientation takes place. It is based on the DMS information about the space position of the mounted object. The basis of the automatic mode consists of the algorithms for the panel position corrections during its installation. The process of the panel erection is carried out with provision for wind loads. The pre-assigned accuracy of positioning at substantial wind loads is obtained at the expense of applying adaptive algorithms of positioning and erecting the panels. Application of the adaptive control for the positioning robot has made it possible to restrict substantially forces in gripping devices.

Planning the trajectory of the mounted panel movement is performed in joint coordinates of the crane $\mathbf{g}_{c}(t)$. Simulation of the erecting operation is carried out on the grounds of the DMS information and the parameters of the system conditions. As a result of simulation the controlling moments of the positioning robot are determined. A specific feature of the system control is the availability of surplus communication; as a consequence there is a multiplicity of solutions satisfying the movement along the pre-set trajectory. To ensure unambiguity the solution the joint moments are determined on the basis of applying standard control actions in the model. Simulation is performed in real time on the grounds of the measured wind load. The force in the places of vacuum grippers attachments serves as a control quantity. For the purpose of control we suggest to use a continuous proportionally differentiating controller ensuring necessary dynamic and precision characteristics described by the equation of the kind

$$
\begin{aligned}
& \mathbf{N}^{(k)}=\left(\delta_{m}+\gamma_{m}\right)\left(T_{c}\left(\mathbf{g}_{c}\right)-T_{c}\left(\mathbf{g}_{m}\right)\right) \cdot \mathbf{d} \mathbf{x}_{c}^{(k)} \\
& , k=\overline{1, l},
\end{aligned}
$$

where $\delta_{m}, \gamma_{m}$ are the coefficients of the proportional and differentiating component of control; $\mathbf{g}_{m}$ are the joint variables of the crane model.The desired distribution of load between the suction cups of the gripper can be obtained by the way of choosing the regulator's coefficients. In order to limit forces in suction cups the system of equations (6) is supplemented with inequalities $\mathrm{N}^{(k)} \leq \mathrm{N}_{\text {lim }}^{(k)}$.

The trajectory of the positioning robot is determined in terms of the non-linear equation

$$
T_{c}\left(\mathrm{~g}_{m}\right) \cdot \mathrm{dx} \mathrm{x}_{c}^{(k)}=T_{r}\left(\mathrm{~g}_{r}\right) \cdot \mathrm{dx}_{r}^{(k)} .
$$

Feedback with real object is implemented by introducing the model parameters correction loop. Measurements of the crane joint coordinates are carried out in discrete moments of time at interval of $\Delta t$. Introducing into the system of dynamic equations additional summands performs the model parameters correction

$$
\begin{aligned}
& \ddot{\mathrm{g}}_{m}=D_{m}\left(\mathrm{~g}_{m}\right) \cdot\left(\begin{array}{l}
\mathrm{M}_{m}-\mathrm{h}_{m}\left(\mathrm{~g}_{m}, \dot{\mathrm{g}}_{m}\right)- \\
-\mathrm{c}_{m}\left(\mathrm{~g}_{m}\right)-\sum_{k=1}^{l} \mathrm{M}_{b}^{(k)}
\end{array}\right), \\
& \dot{\mathrm{g}}_{m}=\int_{0}^{t}\left(\ddot{\mathrm{g}}_{m}+\Delta \ddot{\mathrm{g}}_{m}\right) d \tau, \\
& \mathrm{g}_{m}=\int_{0}^{t}\left(\dot{\mathrm{g}}_{m}+\Delta \dot{\mathrm{g}}_{m}\right) d \tau,
\end{aligned}
$$

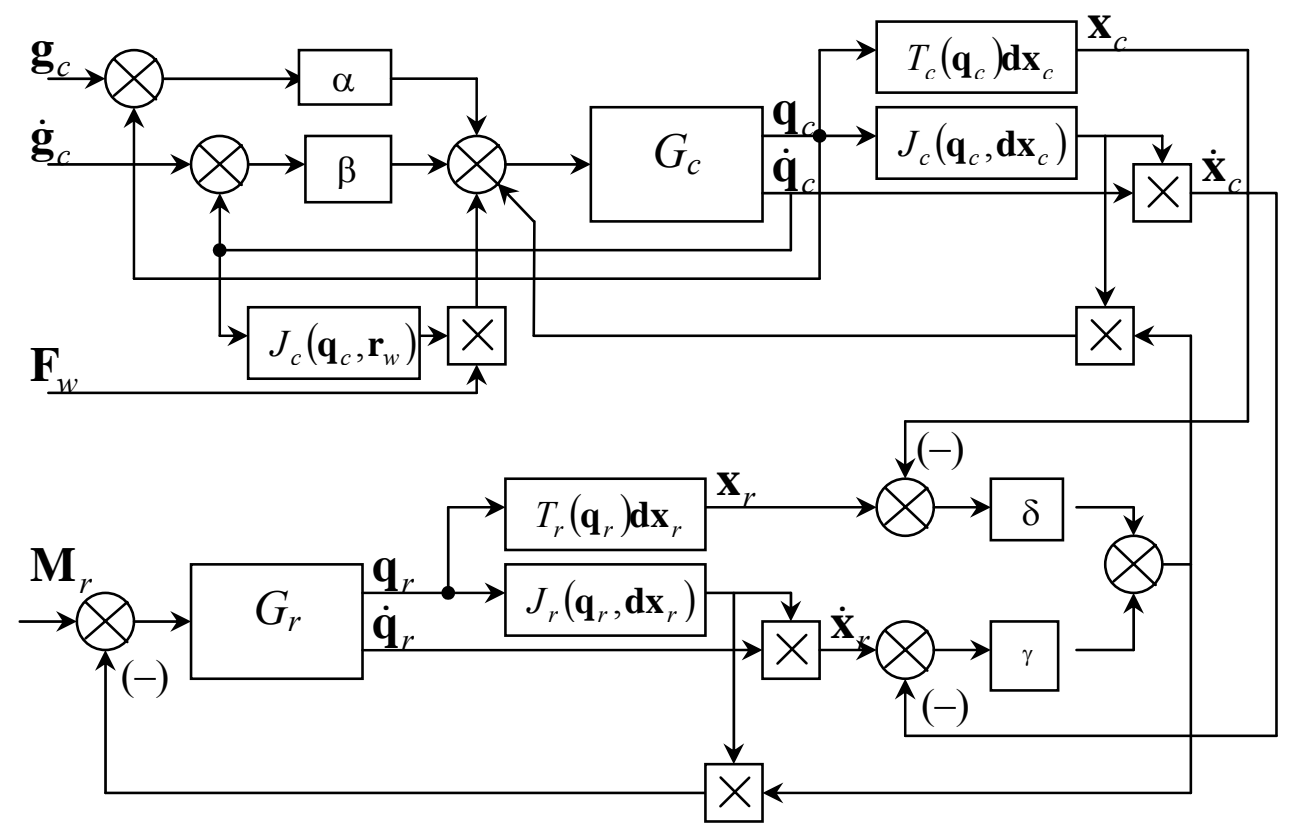

Fig. 2. 
Additional summands are determined by the difference of calculated and measured values of the crane kinematic parameters

$$
\begin{aligned}
& \Delta \dot{\mathrm{g}}_{m}=\left(\mathrm{g}_{m}-\mathrm{q}_{c}\right) \cdot \kappa_{\mathrm{q}}, \\
& \Delta \ddot{\mathrm{g}}_{m}=\left(\dot{\mathrm{g}}_{m}-\mathrm{q}_{c}\right) \cdot \kappa_{\dot{\mathrm{q}}},
\end{aligned}
$$

where $\mathrm{q}_{c}[I \cdot \Delta t], \dot{\mathrm{q}}_{c}[I \cdot \Delta t]$ are the position and the speed of the crane joint coordinates which are measured by an observer; $\kappa_{\mathrm{q}}, \kappa_{\dot{q}}$ are the observer convergency coefficients; $I$ is the number of the current measurement.Calculation of the joint forces moments necessary to overcome the robot inertia while going along the trajectory $\mathrm{g}_{r}(t)$ is performed in terms of the calculation method of controlling moments [1].

Applying the stated procedure of the positioning robot control the system operation simulation has been carried out. In fig. 3 one can see the plots of position

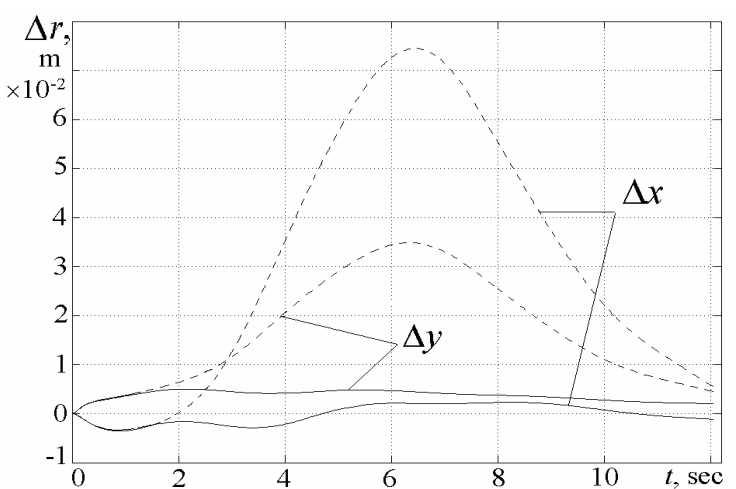

- without robot;

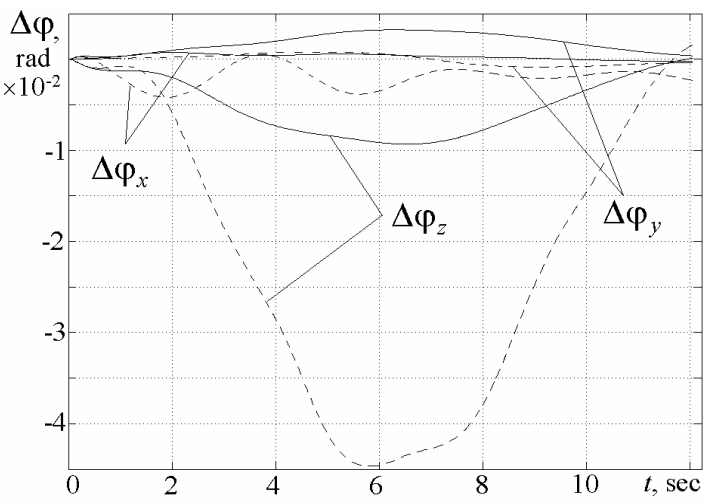

- with robot.

Fig. 3.

change and panel orientation during the process of its erection by the positioning robot at wind load. To compare it represents the passage of the erection trajectory by the crane without incorporation of the positioning robot. The investigations have shown that the imposition of constraints of the positioning robot allowed to increase substantially erection speed and the application of adaptive control has provided the system stability to changing wind load.

\section{CONCLUSION}

The matter for the paper has been prepared in terms of the author's investigations being carried on in the field of robotization of erection works. The presented principles of constructing robotic mounting system are supposed to apply when developing projects for robotization of erection work in the large-panel house-building. The investigations have shown that introduction of the positioning robot into the mounting system substantially increased speed and reliability.

\section{REFERENCES}

[1] Fu K. S., Gonzalez R. C., Lee C. S. G. Robotics: Control, Sensing, Vision, and Intelligence. - McGraw - Hill Company, New York, 1987. 